\title{
FlowPools: A Lock-Free Deterministic Concurrent Dataflow Abstraction
}

\author{
Aleksandar Prokopec ${ }^{1}$, Heather Miller ${ }^{1}$, Tobias Schlatter ${ }^{1}$, Philipp Haller ${ }^{2}$, and \\ Martin Odersky ${ }^{1}$ \\ 1 EPFL, Switzerland \\ firstname.lastname@epfl.ch \\ 2 Typesafe, Inc. \\ firstname.lastname@typesafe.com
}

\begin{abstract}
Implementing correct and deterministic parallel programs is challenging. Even though concurrency constructs exist in popular programming languages to facilitate the task of deterministic parallel programming, they are often too low level, or do not compose well due to underlying blocking mechanisms. In this paper, we present the design and implementation of a fundamental data structure for composable deterministic parallel dataflow computation through the use of functional programming abstractions. Additionally, we provide a correctness proof, showing that the implementation is linearizable, lock-free, and deterministic. Finally, we show experimental results which compare our FlowPool against corresponding operations on other concurrent data structures, and show that in addition to offering new capabilities, FlowPools reduce insertion time by $49-54 \%$ on a 4 -core i7 machine with respect to comparable concurrent queue data structures in the Java standard library.
\end{abstract}

Keywords: dataflow, concurrent data-structure, deterministic parallelism

\section{Introduction}

Multicore architectures have become ubiquitous- even most mobile devices now ship with multiple core processors. Yet parallel programming has yet to enter the daily workflow of the mainstream developer. One significant obstacle is an undesirable choice programmers must often face when solving a problem that could greatly benefit from leveraging available parallelism. Either choose a nondeterministic, but performant, data structure or programming model, or sacrifice performance for the sake of clarity and correctness.

Programming models based on dataflow $[1,2]$ have the potential to simplify parallel programming, since the resulting programs are deterministic. Moreover, dataflow programs can be expressed more declaratively than programs based on mainstream concurrency constructs, such as shared-memory threads and locks, as programmers are only required to specify data and control dependencies. This allows one to reason sequentially about the intended behavior of their program, meanwhile enabling the underlying framework to effectively extract parallelism. 
In this paper, we present the design and implementation of FlowPools, a fundamental dataflow collections abstraction which can be used as a building block for larger and more complex deterministic and parallel dataflow programs. Our FlowPool abstraction is backed by an efficient non-blocking data structure. As a result, our data structure benefits from the increased robustness provided by lock-freedom [12], since its operations are not blocked by delayed threads. We provide a lock-freedom proof, which guarantees progress regardless of the behavior, including the failure, of concurrent threads.

In combining lock-freedom with a functional interface, we go on to show that FlowPools are composable. That is, using prototypical higher-order functions such as foreach and aggregate, one can concisely form dataflow graphs, in which associated functions are executed asynchronously in a completely nonblocking way, as elements of FlowPools in the dataflow graph become available.

Finally, we show that FlowPools are able to overcome practical issues, such as out-of-memory errors, thus enabling programs based upon FlowPools to run indefinitely. By using a builder abstraction, instead of something like iterators or streams (which can lead to non-determinism) we are able to garbage collect parts of the data structure we no longer need, thus reducing memory consumption.

Our contributions are the following:

1. The design and Scala [19] implementation ${ }^{3}$ of a parallel dataflow abstraction and underlying data structure that is deterministic, lock-free, \& composable.

2. Proofs of lock-freedom, linearizability, and determinism.

3. Detailed benchmarks comparing the performance of our FlowPools against other popular concurrent data structures.

\section{Model of Computation}

FlowPools are similar to a typical collections abstraction. Operations invoked on a FlowPool are executed on its individual elements. However, FlowPools do not only act as a data container of elements. Unlike a typical collection, FlowPools also act as nodes and edges of a directed acyclic computation graph (DAG), in which the executed operations are registered with the FlowPool.

Nodes in this directed acyclic graph are data containers which are first class values. This makes it possible to use FlowPools as function arguments or to receive them as return values. Edges, on the other hand, can be thought of as combinators or higher-order functions whose user-defined functions are the previously-mentioned operations that are registered with the FlowPool. In addition to providing composability, this means that the DAG does not have to be specified at compile time, but can be generated dynamically at run time instead.

This structure allows for complete asynchrony, allowing the runtime to extract parallelism as a result. That is, elements can be asynchronously inserted, all registered operations can be asynchronously executed, and new operations can be asynchronously registered. Put another way, invoking several higher-order functions in succession on a given FlowPool does not add barriers between nodes in

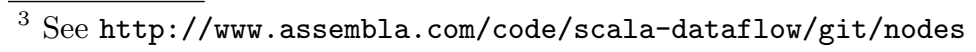


the DAG, it only extends the DAG. This means that individual elements within a FlowPool can flow through different edges of the DAG independently.

Properties of FlowPools. In our model, FlowPools have certain properties which ensure that resulting programs are deterministic.

1. Single-assignment - an element added to the FlowPool cannot be removed.

2. No order - data elements in FlowPools are unordered.

3. Purity - traversals are side-effect free (pure), except when invoking FlowPool operations.

4. Liveness - callbacks are eventually asynchronously executed on all elements.

We claim that FlowPools are deterministic in the sense that all execution schedules either lead to some form of non-termination (e.g., some exception), or the program terminates and no difference can be observed in the final state of the resulting data structures. This definition is practically useful, because in the case of non-termination it is guaranteed that on some thread an exception is thrown which aids debugging, e.g., by including a stack trace. For a more formal definition and proof of determinism, see section 5 .

\section{Programming Interface}

A FlowPool can be thought of as a concurrent pool data structure, i.e., it can be used similarly to a collections abstraction, complete with higher-order functions, or combinators, for composing computations on FlowPools. In this section, we describe the semantics of several of those functional combinators and other basic operations defined on FlowPools.

Append $(<<)$. The most fundamental of all operations on FlowPools is the concurrent thread-safe append operation. As its name suggests, it simply takes an argument of type Elem and appends it to a given FlowPool.

Foreach and Aggregate. A pool containing a set of elements is of little use if its elements cannot be manipulated in some manner. One of the most basic data structure operations is element traversal, often provided by iterators or streams- stateful objects which store the current position in the data structure. However, since their state can be manipulated by several threads at once, using streams or iterators can result in nondeterministic executions.

Another way to traverse the elements is to provide a higher-order foreach operator which takes a user-specified function as an argument and applies it to every element. For it to be deterministic, it must be called for every element that is eventually inserted into the FlowPool, rather than only on those present when foreach is called. Furthermore, determinism still holds even if the user-specified function contains side-effecting FlowPool operations such as $<<$. For foreach to be non-blocking, it cannot wait until additional elements are added to the FlowPool. Thus, the foreach operation must execute asynchronously, and be eventually applied to every element. Its signature is def foreach $[U](f: T \Rightarrow U)$ : Future [Int], and its return type Future[Int] is an integer value which becomes available once foreach traverses all the elements added to the pool. This integer denotes the number of times the foreach has been called. 
The aggregate operation aggregates the elements of the pool and has the following signature: def aggregate $[S]$ (zero: $=>S)(c b:(S, S) \Rightarrow S$ ) (op: $(\mathrm{S}, \mathrm{T}) \Rightarrow \mathrm{S}$ ) : Future $[\mathrm{S}]$, where zero is the initial aggregation, $\mathrm{cb}$ is an associative operator which combines several aggregations, op is an operator that adds an element to the aggregation, and Future [S] is the final aggregation of all the elements which becomes available once all the elements have been added. The aggregate operator divides elements into subsets and applies the aggregation operator op to aggregate elements in each subset starting from the zero aggregation, and then combines different subset aggregations with the $\mathrm{cb}$ operator. In essence, the first part of aggregate defines the commutative monoid and the functions involved must be non-side-effecting. In contrast, the operator op is guaranteed to be called only once per element and it can have side-effects.

While in an imperative programming model, foreach and aggregate are equivalent in the sense that one can be implemented in terms of the other, in a single-assignment programming model aggregate is more expressive. The foreach operation can be implemented using aggregate, but not vice versa.

Builders. The FlowPool described so far must maintain a reference to all the elements at all times to implement the foreach operation correctly. Since elements are never removed, the pool may grow indefinitely and run out of memory. However, it is important to note that appending new elements does not necessarily require a reference to any of the existing elements. This observation allows us to move the $<$ operation out of the FlowPool and into a different abstraction called a builder. Thus, a typical application starts by registering all the foreach operations, and then it releases the references to FlowPools, leaving only references to builders. In a managed environment, the GC then can automatically discard the no longer needed objects.

Seal. After deciding that no more elements will be added, further appends can be disallowed by calling seal. This has the advantage of discarding the registered foreach operations. More importantly, the aggregate can complete its future- this is only possible once it is known there will be no more appends.

Simply preventing append calls after the point when seal is called, however, yields a nondeterministic programming model. Imagine a thread that attempts to seal the pool executing concurrently with a thread that appends an element. In one execution, the append can precede the seal, and in the other the append can follow the seal, causing an error. To avoid nondeterminism, there has to be an agreement on the current state of the pool. A convenient and sufficient way to make seal deterministic is to provide the expected pool size as an argument. The semantics of seal is such that it fails if the pool is already sealed with a different size or the number of elements is greater than the desired size. Note that we do not guarantee that the same exception always occurs on the same thread-rather, if any thread throws some exception in some execution schedule, then in all execution schedules some thread will throw some exception.

Higher-order operators. We now show how these basic abstractions can be used to build higher-order abstractions. To start, it is convenient to have generators that create certain pool types. In a dataflow graph, FlowPools created 


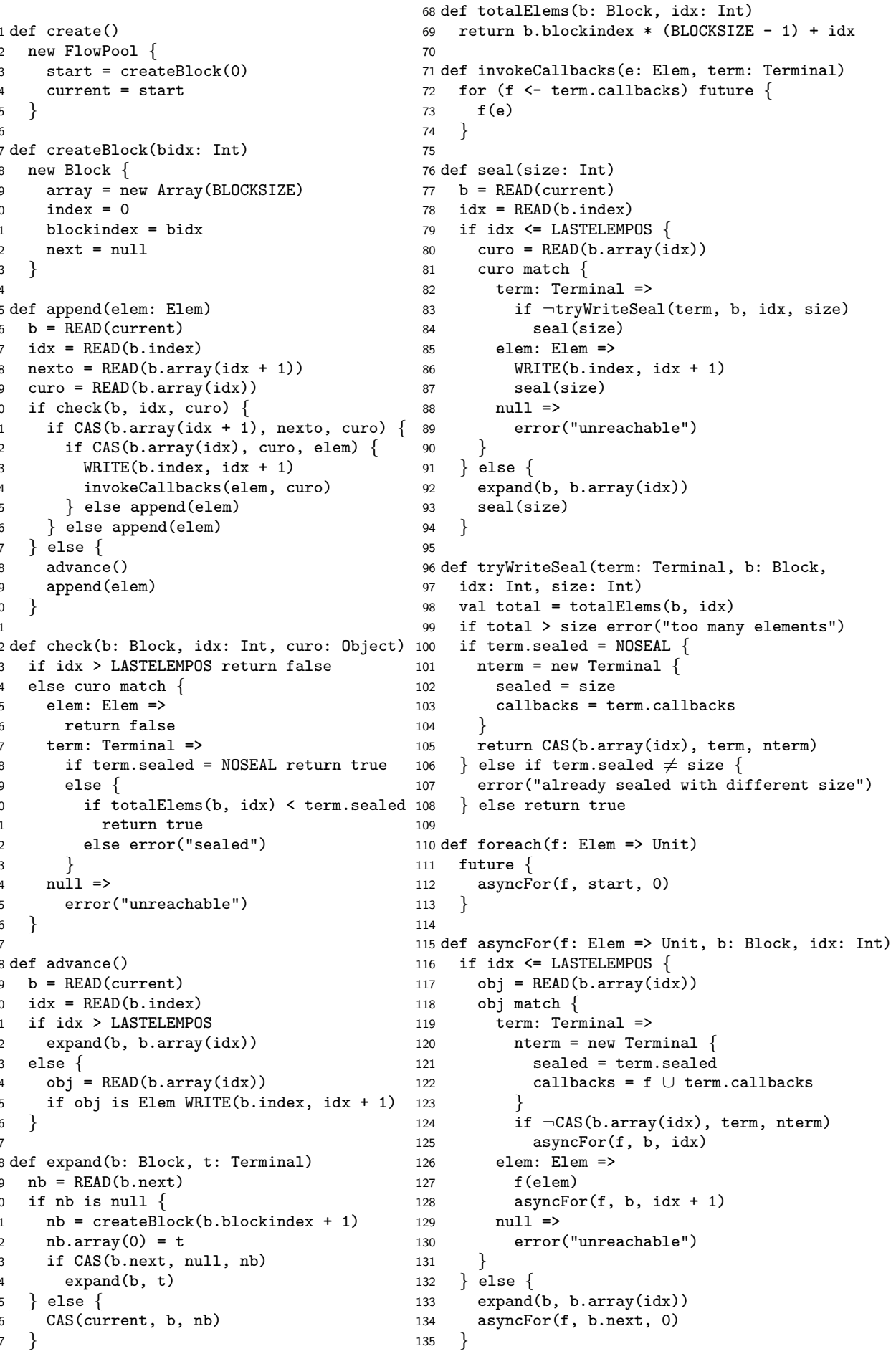

Fig. 2. FlowPool operations pseudocode 
The append operation starts by reading the current block and the index of the free position. It then reads nexto after the first free entry, followed by a read of the curo at the free entry. The check procedure checks the conditions of the bounds, whether the FlowPool was already sealed or if the current array entry contains an element. In either of these events, the current and index values need to be set- this is done in the advance procedure. We call this the slow path of the append method. Notice that there are several situations which trigger the slow path. For example, if some other thread completes the append method but is preempted before updating the value of the hint index, then the curo will have the type Elem. The same happens if a preempted thread updates the value of the hint index after additional elements have been added, via unconditional write in line 23. Finally, reaching an end of block triggers the slow path.

Otherwise, the operation executes the fast path and appends an element. It first copies the Terminal value to the next entry with a CAS instruction in line 21 , with nexto being the expected value. If it fails (e.g. due to a concurrent CAS), the append operation is restarted. Otherwise, it proceeds by writing the element to the current entry with a CAS in line 22 , the expected value being curo. On success, it updates the $\mathrm{b}$. index value and invokes all the callbacks (present when the element was added) with the future construct. In the implementation, we do not schedule an asynchronous computation for each element. Instead, the callback invocations are batched to avoid the scheduling overhead- the array is scanned for new elements until the first free entry is reached.

Interestingly, note that inverting the order of the reads in lines 18 and 19 would cause a race in which a thread could overwrite a Terminal value with some older Terminal value if some other thread appended an element in between.

The seal operation continuously increases the index in the block until it finds the first free entry. It then tries to replace the Terminal value there with a new Terminal value which has the seal size set. An error occurs if a different seal size is set already. The foreach operation works in a similar way, but is executed asynchronously. Unlike seal, it starts from the first element in the pool and calls the callback for each element until it finds the first free entry. It then replaces the Terminal value with a new Terminal value with the additional callback. From that point on the append method is responsible for scheduling that callback for subsequently added elements. Note that all three operations call expand to add an additional block once the current block is empty, to ensure lock-freedom.

Multi-Lane FlowPools. Using a single block sequence (i.e. lane) to implement a FlowPool does not take full advantage of the lack of ordering guarantees and may cause slowdowns due to collisions when multiple concurrent writers are present. Multi-Lane FlowPools overcome this limitation by having a lane for each CPU, where each lane has the same implementation as the normal FlowPool.

This has several implications. First of all, CAS failures during insertion are avoided to a high extent and memory contention is decreased due to writes occurring in different cache-lines. Second, aggregate callbacks are added to each lane individually and aggregated once all of them have completed. Finally, seal needs to be globally synchronized in a non-blocking fashion. 
Once seal is called, the remaining free slots are split amongst the lanes equally. If a writer finds that its lane is full, it writes to some other lane instead. This raises the frequency of CAS failures, but in most cases happens only when the FlowPool is almost full, thus ensuring that the append operation scales.

\section{Correctness}

We give an outline of the correctness proof here. More formal definitions, and a complete set of lemmas and proofs can be found in the tech report [20].

We define the notion of an abstract pool $\mathbb{A}=($ elems, callbacks, seal $)$ of elements in the pool, callbacks and the seal size. Given an abstract pool, abstract pool operations produce a new abstract pool. The key to showing correctness is to show that an abstract pool operation corresponds to a FlowPool operation- that is, it produces a new abstract pool corresponding to the state of the FlowPool after the FlowPool operation has been completed.

Lemma 5.1 Given a FlowPool consistent with some abstract pool, CAS instructions in lines 21, 63 and 66 do not change the corresponding abstract pool.

Lemma 5.2 Given a FlowPool consistent with an abstract pool (elems, cbs, seal), a successful CAS in line 22 changes it to the state consistent with an abstract pool $(\{$ elem $\} \cup$ elems, cbs, seal $)$. There exists a time $t_{1} \geq t_{0}$ at which every callback $f \in c b s$ has been called on elem.

Lemma 5.3 Given a FlowPool consistent with an abstract pool (elems, cbs, seal), a successful CAS in line 124 changes it to the state consistent with an abstract pool (elems, $(f, \emptyset) \cup c b s$, seal $)$ There exists a time $t_{1} \geq t_{0}$ at which $f$ has been called for every element in elems.

Lemma 5.4 Given a FlowPool consistent with an abstract pool (elems, cbs, seal), a successful CAS in line 105 changes it to the state consistent with an abstract pool (elems, cbs, s), where either seal $=-1 \wedge s \in \mathbb{N}_{0}$ or seal $\in \mathbb{N}_{0} \wedge s=$ seal.

Theorem 5.5 [Safety] Operations append, foreach and seal are consistent with the abstract pool semantics.

Theorem 5.6 [Linearizability] Operations append and seal are linearizable.

Lemma 5.7 After invoking a FlowPool operation append, seal or foreach, if a non-consistency changing CAS in lines 21, 63, or 66 fails, they must have already been completed by another thread since the FlowPool operation began.

Lemma 5.8 After invoking a FlowPool operation append, seal or foreach, if a consistency changing CAS in lines 22, 105, or 124 fails, then some thread has successfully completed a consistency changing CAS in a finite number of steps.

Lemma 5.9 After invoking a FlowPool operation append, seal or foreach, a consistency changing instruction will be completed after a finite number of steps. 


\begin{tabular}{|c|c|c|}
\hline \multirow{2}{*}{\multicolumn{3}{|c|}{$\begin{array}{l}p \in\{(v s, \sigma, c b s) \mid v s \subseteq \text { Elem }, \sigma \in\{-1\} \cup \mathbb{N}, \\
\text { cbs } \subset \text { Elem } \Rightarrow \text { Unit }\}\end{array}$}} \\
\hline & & \\
\hline$p<<v$ & append & $v \in$ Elem \\
\hline $\begin{array}{l}p \text { foreach } f \\
p \text { seal } n\end{array}$ & $\begin{array}{l}\text { foreach } \\
\text { seal }\end{array}$ & $\begin{array}{l}f \in \text { Elem } \Rightarrow \text { Unit } \\
n \in \mathbb{N}\end{array}$ \\
\hline$t_{1} ; t_{2}$ & sequence & \\
\hline
\end{tabular}

Fig. 3. Syntax

Theorem 5.10 [Lock-freedom] FlowPool operations append, foreach and seal are lock-free.

Determinism. We claim that the FlowPool abstraction is deterministic in the sense that a program computes the same result (possibly an error) regardless of the interleaving of execution steps. Here we give an outline of the determinism proof. A complete formal proof can be found in the technical report [20].

The following definitions and the determinism theorem are based on the language shown in Figure 3. The semantics of our core language is defined using reduction rules which define transitions between execution states. An execution state is a pair $T \mid P$ where $T$ is a set of concurrent threads and $P$ is a set of FlowPools. Each thread executes a term of the core language (typically a sequence of terms). State of a thread is represented as the (rest of) the term that it still has to execute; this means there is a one-to-one mapping between threads and terms. For example, the semantics of append is defined by the following reduction rule (a complete summary of all the rules can be found in the appendix):

$$
\frac{t=p<<v ; t^{\prime} \quad p=(v s, c b s,-1) \quad p^{\prime}=(\{v\} \cup v s, c b s,-1)}{t, T\left|p, P \longrightarrow t^{\prime}, T\right| p^{\prime}, P} \text { (APPEnd1) }
$$

Append simply adds the value $v$ to the pool $p$, yielding a modified pool $p^{\prime}$. Note that this rule can only be applied if the pool $p$ is not sealed (the seal size is -1 ). The rule for foreach modifies the set of callback functions in the pool:

$$
\begin{gathered}
t=p \text { foreach } f ; t^{\prime} \quad p=(v s, c b s, n) \\
\frac{T^{\prime}=\{g(v) \mid g \in\{f\} \cup \operatorname{cbs}, v \in v s\} \quad p^{\prime}=(v s,\{f\} \cup c b s, n)}{t, T\left|p, P \longrightarrow t^{\prime}, T, T^{\prime}\right| p^{\prime}, P} \text { (Foreach2) }
\end{gathered}
$$

This rule only applies if $p$ is sealed at size $n$, meaning that no more elements will be appended later. Therefore, an invocation of the new callback $f$ is scheduled for each element $v$ in the pool. Each invocation creates a new thread in $T^{\prime}$.

Programs are built by first creating one or more FlowPools using create. Concurrent threads can then be started by (a) appending an element to a FlowPool, (b) sealing the FlowPool and (c) registering callback functions (foreach).

Definition 5.11 [Termination] A term $t$ terminates with result $P$ if its reduction ends in execution state $\{t: t=\{\epsilon\}\} \mid P$. 
Definition 5.12 [Interleaving] Consider the reduction of a term $t: T_{1} \mid P_{1} \longrightarrow$ $T_{2}\left|P_{2} \longrightarrow \ldots \longrightarrow\{t: t=\{\epsilon\}\}\right| P_{n}$. An interleaving is a reduction of $t$ starting in $T_{1} \mid P_{1}$ in which reduction rules are applied in a different order.

Definition 5.13 [Determinism] The reduction of a term $t$ is deterministic iff either (a) $t$ does not terminate for any interleaving, or (b) $t$ always terminates with the same result for all interleavings.

Theorem 5.14 [FlowPool Determinism] Reduction of terms $t$ is deterministic.

\section{Evaluation}

We evaluate our implementation (single-lane and multi-lane FlowPools) against the LinkedTransferQueue [14] for all benchmarks and the ConcurrentLinkedQueue [17] for the insert benchmark, both found in JDK 1.7, on three different architectures; a quad-core $3.4 \mathrm{GHz}$ i7-2600, 4x octa-core $2.27 \mathrm{GHz}$ Intel Xeon x7560 (both with hyperthreading) and an octa-core $1.2 \mathrm{GHz}$ UltraSPARC T2 with 64 hardware threads. In this section, we focus on the scaling properties of the abovementioned data structures, Figures 4 \& 5 .

In the Insert benchmark, Figure 4, we evaluate concurrent insert operations, by distributing the work of inserting $N$ elements into the data structure concurrently across $P$ threads. In Figure 4, it's evident that both single-lane FlowPools and concurrent queues do not scale well with the number of concurrent threads, particularly on the i7 architecture. They quickly slow down, likely due to cache line collisions and CAS failures. On the other hand, multi-lane FlowPools scale well, as threads write to different lanes, and hence different cache lines, meanwhile also avoiding CAS failures. This appears to reduce execution time for insertions up to $54 \%$ on the i7, $63 \%$ on the Xeon and $92 \%$ on the UltraSPARC.

The performance of higher-order functions is evaluated in the Reduce, Map (both in Figure 4) and Histogram benchmarks (Figure 5). It's important to note that the Histogram benchmark serves as a "real life" example, which uses both the map and reduce operations that are benchmarked in Figure 4. Also note that in all of these benchmarks, the time it takes to insert elements into the FlowPool is also measured, since the FlowPool programming model allows one to insert elements concurrently with the execution of higher-order functions.

In the Histogram benchmark, Figure 5, $P$ threads produce a total of $N$ elements, adding them to the FlowPool. The aggregate operation is then used to produce 10 different histograms concurrently with a different number of bins. Each separate histogram is constructed by its own thread (or up to $P$, for multi-lane FlowPools). A crucial difference between queues and FlowPools here, is that with FlowPools, multiple histograms are produced by invoking several aggregate operations, while queues require writing each element to several queues- one for each histogram. Without additional synchronization, reading a single queue is not an option, since elements have to be removed from the queue eventually, and it is not clear to each reader when to do this. With FlowPools, elements are automatically garbage collected when no longer needed. 
Operations on FlowPools Across Architectures

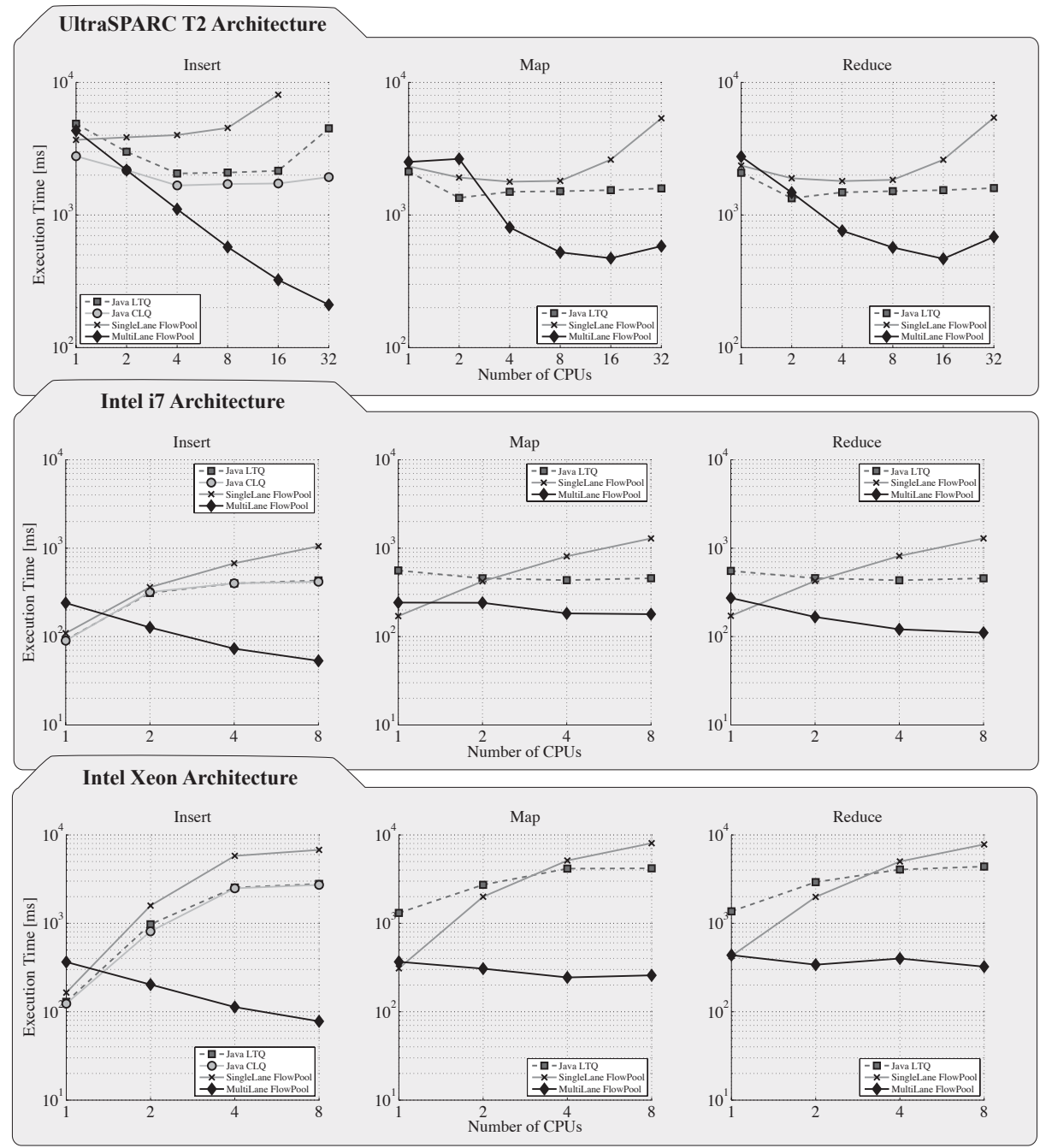

Fig. 4. Execution time vs parallelization across three different architectures on three important FlowPool operations; insert, map, reduce.

Finally, to validate the last claim of garbage being automatically collected, in the Communication/Garbage Collection benchmark, Figure 5, we create a pool in which a large number of elements $N$ are added concurrently by $P$ threads. Each element is then processed by one of $P$ threads through the use of the aggregate operation. We benchmark against linked transfer queues, where $P$ threads concurrently remove elements from the queue and process it. For each run, we vary the size of the $N$ and examine its impact on the execution time. Especially in the cases of the Intel architectures, the multi-lane FlowPools perform considerably better than the linked transfer queues. As a matter of fact, the linked transfer queue on the Xeon benchmark ran out of memory, and was un- 

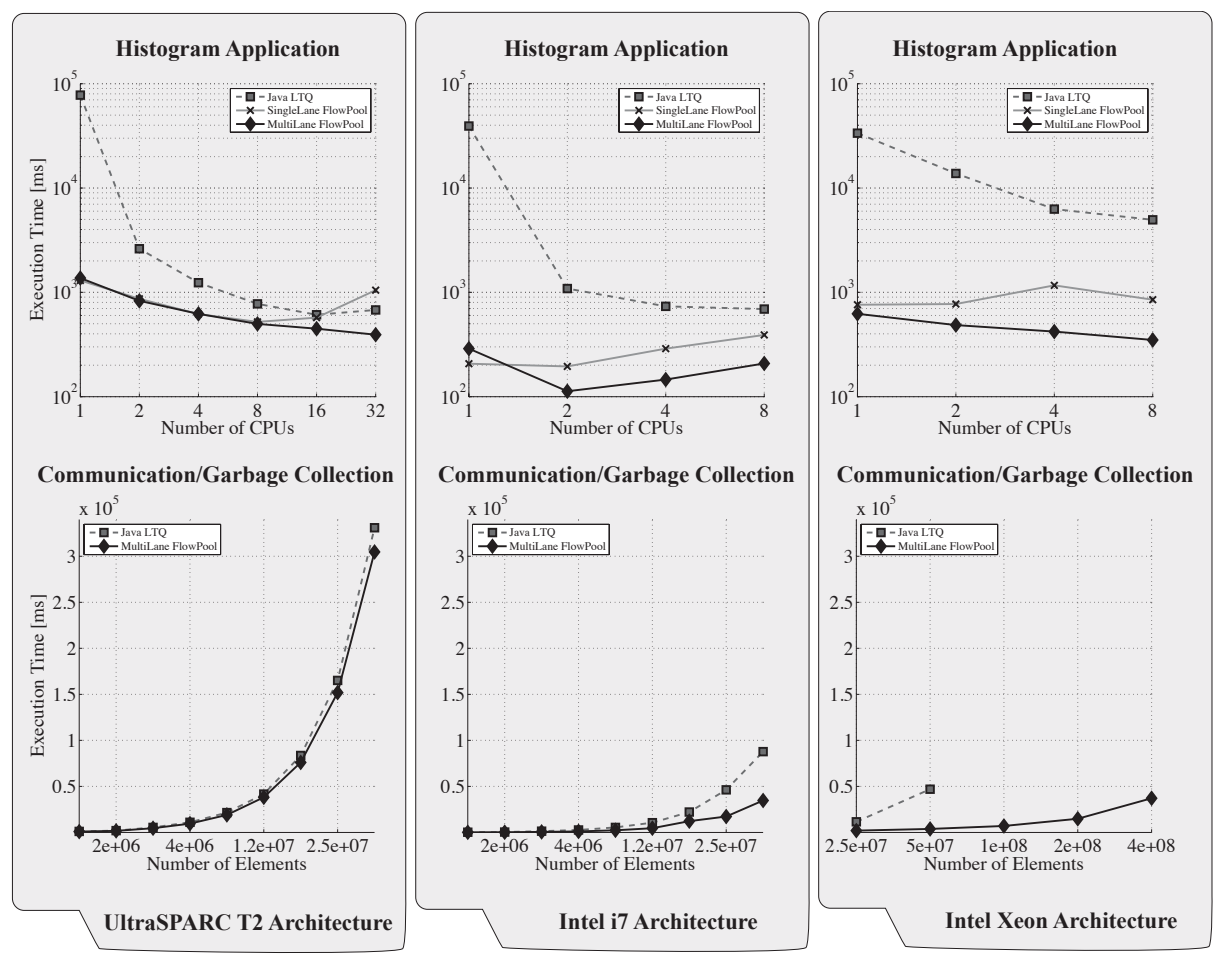

Fig. 5. Execution time vs parallelization on a real histogram application (top), \& communication benchmark (bottom) showing memory efficiency, across all architectures.

able to complete, while the multi-lane FlowPool scaled effortlessly to 400 million elements, indicating that unneeded elements are properly garbage collected.

\section{Related Work}

An introduction to linearizability and lock-freedom is given by Herlihy and Shavit [13]. A detailed overview of concurrent data structures is given by Moir and Shavit [18]. To date, concurrent data structures remain an active area of research- we restrict this summary to those relevant to this work.

Concurrently accessible queues have been present for a while, an implementation is described by [16]. Non-blocking concurrent linked queues are described by Michael and Scott [17]. This CAS-based queue implementation is cited and used widely today, a variant of which is present in the Java standard library. More recently, Scherer, Lea and Scott [14] describe synchronous queues which internally hold both data and requests. Both approaches above entail blocking (or spinning) at least on the consumer's part when the queue is empty.

While the abstractions above fit well in the concurrent imperative model, they have the disadvantage that the programs written using them are inherently nondeterministic. Roy and Haridi [21] describe the Oz programming language, a subset of which yields programs deterministic by construction. Oz dataflow 
streams are built on top of single-assignment variables and are deterministically ordered. They allow multiple consumers, but only one producer at a time. $\mathrm{Oz}$ has its own runtime which implements blocking using continuations.

The concept of single-assignment variables is used to provide logical variables in concurrent logic programming languages [23]. It is also embodied in futures proposed by Baker and Hewitt [11], and promises first mentioned by Friedman and Wise [7]. Futures were first implemented in MultiLISP [10], and have been employed in many languages and frameworks since. Scala 2.10 futures [9] and Twitter futures [6] are of interest, because they define monadic operators and a number of high-level combinators that create new futures. These APIs avoid blocking. Futures have been generalized to data-driven futures, which provide additional information to the scheduler [24]. Many frameworks have constructs that start an asynchronous computation and yield a future holding its result, for example, Habanero Java [3] (async) and Scala [19] (future).

A number of other models and frameworks recognized the need to embed the concept of futures into other data-structures. Single-assignment variables have been generalized to I-Structures [1] which are essentially single-assignment arrays. $\mathrm{CnC}[4,2]$ is a parallel programming model influenced by dynamic dataflow, stream-processing and tuple spaces [8]. In $\mathrm{CnC}$ the user provides high-level operations along with the ordering constraints that form a computation dependency graph. FlumeJava [5] is a distributed programming model which relies heavily on the concept of collections containing futures. An issue that often arises with dataflow programming models are unbalanced loads. This is often solved using bounded buffers which prevent the producer from overflowing the consumer.

Opposed to the correct-by-construction determinism described thus far, a type-systematic approach can also ensure that concurrent executions have deterministic results. Recently, work on Deterministic Parallel Java showed that a region-based type system can ensure determinism [15]. X10's constrained-based dependent types can similarly ensure determinism and deadlock-freedom [22].

\section{Conclusion}

The abstraction for concurrent dataflow programming we presented provides a composable deterministic programming model. It can be implemented in a provably non-blocking manner and is efficient as well, as shown in experiments.

As future work, we plan developing other concurrent collection types with deterministic semantics, which enrich the correct-by-construction single-assignment model, such as bounded buffers, streams and maps. On the implementation level, we anticipate the need of embedding the callbacks within the data-structure itself, as is the case with callback-based futures and FlowPools - this has a particular benefit on platforms which do not support efficient continuations.

\section{References}

1. Arvind, R. S. Nikhil, and K. K. Pingali. I-structures: Data structures for parallel computing. ACM Trans. Prog. Lang. and Sys., 11(4):598-632, Oct. 1989. 
2. Z. Budimlic, M. G. Burke, V. Cavé, K. Knobe, G. Lowney, R. Newton, J. Palsberg, D. M. Peixotto, V. Sarkar, F. Schlimbach, and S. Tasirlar. Concurrent collections. Scientific Programming, 18(3-4):203-217, 2010.

3. Z. Budimlic, V. Cavé, R. Raman, J. Shirako, S. Tasirlar, J. Zhao, and V. Sarkar. The design and implementation of the Habanero-Java parallel programming language. In OOPSLA Companion, pages 185-186, 2011.

4. M. G. Burke, K. Knobe, R. Newton, and V. Sarkar. Concurrent collections programming model. In Encyclopedia of Parallel Computing, pages 364-371. 2011.

5. C. Chambers, A. Raniwala, F. Perry, S. Adams, R. R. Henry, R. Bradshaw, and N. Weizenbaum. FlumeJava: easy, efficient data-parallel pipelines. ACM SIGPLAN Notices, 45(6):363-375, June 2010.

6. M. Eriksen and N. Kallen. Twitter Finagle: Futures. See http://twitter.github.com/finagle/.

7. D. Friedman and D. Wise. The impact of applicative programming on multiprocessing. In International Conference on Parallel Processing, 1976.

8. D. Gelernter. Generative communication in Linda. ACM Transactions on Programming Languages and Systems, 7(1):80-112, Jan. 1985.

9. P. Haller, A. Prokopec, H. Miller, V. Klang, R. Kuhn, and V. Jovanovic. Scala improvement proposal: Futures and promises (SIP-14). 2012. See http://docs.scala-lang.org/sips/pending/futures-promises.html.

10. J. R. H. Halstead. MultiLISP: A language for concurrent symbolic computation. ACM Trans. Prog. Lang. and Sys., 7(4):501-538, Oct. 1985.

11. J. Henry C. Baker and C. Hewitt. The incremental garbage collection of processes. In Proc. Symp. on Art. Int. and Prog. Lang., 1977.

12. M. Herlihy. A methodology for implementing highly concurrent data structures. In PPoPP, pages 197-206, 1990.

13. M. Herlihy and N. Shavit. The Art of Multiprocessor Programming. Apr. 2008.

14. W. N. S. III, D. Lea, and M. L. Scott. Scalable synchronous queues. Commun. ACM, 52(5):100-111, 2009.

15. R. L. B. Jr., V. S. Adve, D. Dig, S. V. Adve, S. Heumann, R. Komuravelli, J. Overbey, P. Simmons, H. Sung, and M. Vakilian. A type and effect system for deterministic parallel Java. In OOPSLA, pages 97-116, 2009.

16. J. M. Mellor-Crummey. Concurrent queues: Practical fetch-and- $\Phi$ algorithms. 1987.

17. M. M. Michael and M. L. Scott. Simple, fast, and practical non-blocking and blocking concurrent queue algorithms. In PODC, pages 267-275, 1996.

18. Moir and Shavit. Concurrent data structures. In Mehta and Sahni, editors, Handbook of Data Structures and Applications, Chapman \& Hall/CRC. 2005.

19. M. Odersky, L. Spoon, and B. Venners. Programming in Scala. Artima Press, Mountain View, CA, 2010.

20. A. Prokopec, H. Miller, T. Schlatter, P. Haller, and M. Odersky. Flowpools: A lock-free deterministic concurrent dataflow abstraction- proofs. Technical Report EPFL-REPORT-181098, EPFL, Lausanne, June 2012.

21. P. V. Roy and S. Haridi. Concepts, Techniques, and Models of Computer Programming. MIT Press, 2004.

22. V. A. Saraswat, V. Sarkar, and C. von Praun. X10: concurrent programming for modern architectures. In PPOPP, page 271, 2007.

23. E. Shapiro. The family of concurrent logic programming languages. ACM Computing Surveys, 21(3):412, Sept. 1989.

24. S. Tasirlar and V. Sarkar. Data-driven tasks and their implementation. In $I C P P$, pages 652-661, 2011. 